It is possible to obtain Bailey's identity from either (6) or ( 7 ).

3. Conclusion. This classification of the basic analogues of Thomae's two-term relations indicates, then, that out of the entire set of identities, only two are essentially distinct. Any further results can be obtained from this source only by specialization of the parameters in Hall's identity or in relation (6) or (7).

Analogous statements can be made concerning the corresponding relations between the generalized hypergeometric functions ${ }_{3} F_{2}$ as given by Whipple.

Agricultural and Mechanical College of Texas and

UNIVERSITY OF NEBRASKa

\title{
A NOTE ON FINITE REGULAR RINGS
}

\section{JOHN DYER-BENNET}

The purpose of this note is to give a classification of all finite regular rings. One result of the classification is the extension of a part of Wedderburn's classic theory of the structure of linear associative algebras to systems which are not algebras. I wish to thank Professor Garrett Birkhoff for several suggestions in the preparation of the note.

Definition. $A$ ring $R$ is said to be regular if for every element a of $R$ there exists an element $x$ in $R$ such that

$$
a k a=a .^{1}
$$

\section{Lemma 1. A regular ring has radical $(0){ }^{2}$}

Proof. Since the radical of a ring is a nilpotent ideal, any element $a$ in it has the property that a product containing $a$ as a factor a sufficient number of times is zero. Since by definition there exists an $x$ such that $a x a x \cdots a x a=a$ no matter how many terms there are in the product, zero must be the only member of the radical.

${ }^{1}$ Cf. von Neumann, Lectures on Continuous Geometries, Part 2, Princeton, 1937, pp. 7-21. Also On regular rings, Proceedings of the National Academy of Sciences, vol. 22 (1936), pp. 707-713. von Neumann postulates the existence of a unit, but in our case this follows from the other assumptions.

${ }^{2}$ Cf. von Neumann, loc. cit. 
From Lemma 1 it follows that a regular ring which satisfies the finite descending chain condition for ideals is semi-simple, and hence contains a unit.

DEFINITION. The characteristic of a ring is the least common multiple of the additive orders of its elements, if this is finite, otherwise $\infty$.

If the ring has a unit this is the additive order of the unit, so that this definition agrees with the usual one for a field.

LEMMA 2. The characteristic of a regular ring with a unit is squarefree, if it is finite.

Proof. Suppose the characteristic $c$ is not square-free. Let

$$
c=p_{1}^{e_{1}} p_{2}^{e_{2}} \cdots p_{r}^{e_{r}}
$$

where we may assume that $e_{1}>1$. Let $a=c / p_{1}$ where we are considering $a$ as an element of the ring. Then $a \neq 0$. Further, for any $x$ in the ring

$$
a x a=a a x=0 \neq a .
$$

It follows that the ring is not regular.

ThEOREM 1. A finite regular ring is the direct sum of algebras.

Proof. By the theory of commutative groups the additive group of the ring is the direct sum of subgroups each of which consists of all the elements whose order is a fixed prime $p \cdot{ }^{3}$ Since the order of a product of two elements of order $p$ is also of order $p$-unless the product is zero-these subgroups are also subrings. Next, let $a$ and $b$ be two elements of additive orders $p$ and $q$ respectively, $p \neq q$. Then

$$
p a b=(p a) b=0 \cdot b=0
$$

and

$$
q a b=a(q b)=a \cdot 0=0,
$$

where $p c$ stands for a sum of $p$ summands $c$. Thus the order of $a b$ is less than or equal to $p$ and to $q$, and divides both. Hence $a b=0$. Since the product of any two elements from different subrings is zero, the ring is the direct sum of these subrings. Each of these subrings is of prime characteristic, and hence contains in its center a prime field of $p$ elements. Over this field it is clearly a linear associative algebra. Finally, each of these is clearly semi-simple.

${ }^{3}$ Cf. Speiser, Theorie der Gruppen von endlicher Ordnung, 3d edition, p. 49. 
THEOREM 2. A finite regular ring is the direct sum of full matrix algebras over finite fields, and the summands are unique, apart from their order.

Proof. By Theorem 1 it is the direct sum of semi-simple algebras. By a theorem due to Wedderburn each of these is a direct sum of simple algebras, and these are unique, apart from their order. ${ }^{4}$ But a simple algebra is a total matrix algebra over a division algebra. ${ }^{5}$ Finally, every finite division algebra is commutative, and hence a field. ${ }^{6}$

The proof of Theorem 2 above seems to lend itself to generalization, but it is not the simplest proof that can be given. By relying even more heavily on results in the literature the theorem can be proved as follows.

von Neumann has shown that a regular ring with a unit is irreducible if and only if its center is a field. ${ }^{7}$ Hence a finite regular ring is the direct sum of (finite regular) rings whose centers are fields. The terms in this direct sum are uniquely determined. ${ }^{8}$ This reduces the problem to that of classifying the finite regular rings whose centers are fields. Such a ring is a normal simple linear associative algebra over its center, ${ }^{9}$ therefore a total matric algebra over its center. ${ }^{10}$

There is an immediate corollary of the results above. Since a regular ring is irreducible if and only if its center is a field, there follows the theorem (due to Dedekind ${ }^{11}$ ) that a commutative regular ring satisfying the descending chain condition is the direct sum of fields.

We can also get another proof of what is almost Lemma 2, namely that a semi-simple ring has a square-free characteristic if the characteristic is finite. For the direct sum of two fields of characteristic $p$ is of characteristic $p$, while the direct sum of two fields of characteristics $p$ and $q$ respectively has characteristic $p q$. This result is interesting for the following reason. The regularity condition (1) above is a natural weakening of the condition that inverses should exist, ${ }^{12}$ and so a regular ring might be thought of as somewhere between an arbi-

${ }^{4}$ Albert, Structure of Algebras, American Mathematical Society Colloquium Publications, vol. 24, p. 39.

${ }^{5}$ Cf. Albert, loc. cit., p. 39.

${ }^{6}$ Cf. Albert, loc. cit., p. 62.

7 Continuous Geometries, Part 2, p. 21.

${ }^{8}$ van der Waerden, Moderne Algebra, vol. 2, p. 162.

${ }^{9}$ Albert, loc. cit., pp. 6, 37.

${ }^{10}$ Albert, loc. cit., p. 62.

${ }^{11}$ Cf. van der Waerden, loc. cit., p. 163.

12 Cf. von Neumann, Continuous Geometries, Part 2, p. 21. 
trary ring and a field. Similarly the result that the characteristic is square-free is a natural weakening of the result that the characteristic of a finite field is prime.

We complete the note by giving a combinatory formula for the number of non-isomorphic regular rings of a given finite order. The formula is unsatisfactory in that it involves finding all the partitions of an integer. It does not seem likely, however, that this can be avoided.

The algebra of $n^{2}$ matrices over a field of $p^{k}$ elements has $p^{n^{2} k}$ elements. Hence there are as many total matrix algebras with $p^{e}$ elements as there are squares that divide $e$. Let

$$
e=p_{1}^{e_{1}} \cdots p_{r}^{e_{r}}
$$

Then there are clearly

$$
\prod_{i=1}^{r}\left\{\left[e_{i} / 2\right]+1\right\}
$$

such algebras, where $[a]$ is the greatest integer in $a$. These are all different, since the center of each is isomorphic with the field over which it is taken.

The number of non-isomorphic regular rings, each of which is the direct sum of $s$ simple rings of orders $p^{e i}$ respectively is the product of the numbers for each of the $e_{i}$. The number of order $p^{k}$ is therefore the sum of these products for all possible partitions of $k, k=\sum_{i=1}^{s} e_{i}$.

Using these results and Theorem 2 we see that the number of nonisomorphic rings of order $n=\prod_{t=1}^{r} p_{t}^{k_{t}}$ is

$$
\prod_{t=1}^{r} \sum_{e_{1}+\cdots+e_{s}=k_{t}} \prod_{i=1}^{s} \prod_{j=1}^{r_{i}}\left\{\left[e_{i, j} / 2\right]+1\right\}
$$

where the summation is taken over all partitions of $k_{t}$, and

$$
e_{i}=\prod_{j=1}^{r_{i}} p_{i, j}^{e_{i, j}}
$$

VANDERBILT UNIVERSITY 